FUNCTION SPACES XII

BANACH CENTER PUBLICATIONS, VOLUME 119

INSTITUTE OF MATHEMATICS

POLISH ACADEMY OF SCIENCES

WARSZAWA 2019

\title{
COMPLEX CONVEXITY AND FIXED POINT THEOREMS IN ORLICZ MODULAR SPACES
}

\author{
LILI CHEN \\ College of Mathematics and Systems Science \\ Shandong University of Science and Technology \\ Qingdao 266590, China \\ Department of Mathematics, Harbin University of Science and Technology \\ Harbin 150080, China \\ E-mail: chenlili0819@foxmail.com \\ DEYUN CHEN \\ College of Computer Science and Technology \\ Harbin University of Science and Technology \\ Harbin 150080, China \\ E-mail: 1965197765@qq.com \\ YANG JIANG \\ Department of Mathematics, Harbin University of Science and Technology \\ Harbin 150080, China \\ E-mail: jiangy0418@foxmail.com
}

\begin{abstract}
In this paper, we prove that every Orlicz modular function space $L_{\Phi, \rho}$ is complex midpoint locally uniformly convex. As a corollary, $L_{\Phi, \rho}$ is also complex strictly convex. Furthermore, we introduce the notions of mean nonexpansive mappings in the modular sense and prove a fixed point theorem in Orlicz modular function spaces.
\end{abstract}

2010 Mathematics Subject Classification: Primary 46B20; Secondary 46E30.

Key words and phrases: modular space, complex convexity, $\rho$-mean nonexpansive mapping, fixed point theorem.

The paper is in final form and no version of it will be published elsewhere. 
1. Introduction. In 1967, the notions of complex extreme points and complex strict convexity have been introduced by E. Thorp and R. Whitley [37. They proved that the strong maximum modulus theorem for analytic functions with values in a complex Banach space $X$ holds whenever each point of the unit sphere of $X$ is a complex extreme point (see [36]). In 1975, J. Globevnik further introduced the notions of complex strict and uniform convexity of complex normed spaces and proved that the complex space $L_{1}$ is complex uniformly convex (see [17]). W. Davis, D. Darling and N. TomczakJaegermann in [14] investigated the complex convexity of quasi-normed linear spaces. P. N. Dowling, Z. B. Hu and D. Mupasiri in [15] studied the complex convexity of Lebesgue-Bochner function spaces. O. Blasco and M. Pavlović in [1] obtained sufficient and necessary conditions for a complex Banach space $X$ which is $p$-uniformly PL-convex. C. Choi, A. Kamińska and H. J. Lee in [7] obtained criteria for complex extreme points, complex rotundity and complex uniform convexity in Orlicz-Lorentz spaces. H. Hudzik and A. Narloch in [19] considered relationships between monotonicity and complex rotundity, for instance, a point $f$ of the complexification $E^{C}$ of a real Köthe space $E$ is a complex extreme point if and only if $|f|$ is a point of upper monotonicity in $E$. H. J. Lee in [26, 27] continued studying relationships between monotonicity and complex convexity in Banach lattices and quasi-Banach lattices respectively. M. M. Czerwińska and A. Kamińska in [12] discussed the complex rotundity and midpoint local uniform convexity in symmetric spaces of measurable operators and they also obtained the concepts of complex midpoint local uniform rotundity and complex local uniform rotundity are equivalent for any complex Banach spaces. Recently, M. M. Czerwińska and A. Parrish in 13 characterized complex extreme points in Marcinkiewicz spaces.

In this work, we prove that any Orlicz modular function space $L_{\Phi, \rho}$ is complex midpoint locally uniformly convex. As a corollary, $L_{\Phi, \rho}$ is also complex strictly convex. Furthermore, we introduce the notions of mean nonexpansive mappings in the modular sense and prove a fixed point theorem in Orlicz modular function spaces.

Before starting with our results, we need to recall some basic concepts and facts of the theory of modular spaces and Orlicz spaces. For basic information concerning fixed point theory for nonexpansive mappings see [18].

Let $X$ be a vector space over the complex field $\mathbb{C}$. A functional $\rho: X \rightarrow[0, \infty]$ is called a modular provided that for any $f, g \in X$,

(a) $\rho(f)=0$ if and only if $f=0$;

(b) $\rho(\alpha f)=\rho(f)$ for any $\alpha \in \mathbb{C}$ with $|\alpha|=1$;

(c) $\rho(\alpha f+\beta g) \leq \rho(f)+\rho(g)$ for any $\alpha, \beta \geq 0$ with $\alpha+\beta=1$.

If we replace (c) by

$\left(\mathrm{c}^{\prime}\right) \rho(\alpha f+\beta g) \leq \alpha \rho(f)+\beta \rho(g)$ for any $\alpha, \beta \geq 0$ with $\alpha+\beta=1$,

then the modular $\rho$ is said to be a convex modular. A modular space $X_{\rho}$ is defined by

$$
X_{\rho}=\{f \in X: \rho(\lambda f) \rightarrow 0 \text { as } \lambda \rightarrow 0\} .
$$

Let $X_{\rho}$ be a modular space, then

$$
B\left(X_{\rho}\right)=\left\{x \in X_{\rho}: \rho(x) \leq 1\right\}, \quad \text { and } \quad S\left(X_{\rho}\right)=\left\{x \in X_{\rho}: \rho(x)=1\right\}
$$


denote the closed unit ball and the unit sphere of $X_{\rho}$ respectively. In the sequel $\mathbb{N}, \mathbb{R}$ and $\mathbb{C}$ denote the set of natural numbers, the set of real numbers and the set of complex numbers, respectively. Let $i$ be the complex number satisfying $i^{2}=-1$.

A map $\Phi: R \rightarrow[0, \infty]$ is said to be an Orlicz function if $\Phi$ is vanishing at zero, even, convex and continuous, and satisfies $\lim _{u \rightarrow 0} \frac{\Phi(u)}{u}=0$ and $\lim _{u \rightarrow \infty} \frac{\Phi(u)}{u}=\infty$. For every Orlicz function $\Phi$, its complementary function $\Psi: R \rightarrow[0, \infty]$ is defined by the formula

$$
\Psi(v)=\sup \{u|v|-\Phi(u): u \geq 0\},
$$

the complementary function $\Psi$ is also an Orlicz function.

For an Orlicz function $\Phi$, we define

$$
a_{\Phi}=\max \{u \geq 0: \Phi(u)=0\}, \quad b_{\Phi}=\max \{u \geq 0: \Phi(u)<\infty\} .
$$

Let $(T, \Sigma, \mu)$ be a $\Sigma$-finite, atomless measure space with a complete $\mu$-measure. $L^{o}$ is the family of all $\Sigma$-measurable functions defined on $T$. For a given Orlicz function $\Phi$, the Orlicz modular is defined by the formula

$$
\rho(f)=\int_{T} \Phi(|f(t)|) d \mu \quad \forall f \in L^{o} .
$$

Let $\operatorname{supp}(f)=\{t \in T:|f(t)| \neq 0\}$. The Orlicz space $L_{\Phi}$ is generated by an Orlicz function $\Phi$ by the formula

$$
L_{\Phi}=\left\{f \in L^{o}: \rho(c f)<\infty \text {, for some } c>0 \text { depending on } f\right\} .
$$

$L_{\Phi}$ is usually equipped with the Luxemburg norm

$$
\|f\|_{\Phi}=\inf \left\{\varepsilon>0: \rho\left(\frac{f}{\varepsilon}\right) \leq 1\right\}
$$

or with the equivalent one

$$
\|f\|_{\Phi}^{\circ}=\sup \left\{\int_{T}|f(t) g(t)| d \mu: g \in L_{\Psi}, \rho(g) \leq 1\right\}
$$

called the Orlicz norm.

Indeed, Orlicz spaces and their kinds of generalizations belong to modular spaces. For the sake of simplicity, we define $L_{\Phi, \rho}=\left(L_{\Phi}, \rho\right)$ and $L_{\Phi}=\left(L_{\Phi},\|\cdot\|_{\Phi}\right)$.

Let $X_{\rho}$ be a modular space. Then

(1) We say that $\left(f_{n}\right)$ is $\rho$-convergent to $f$ if $\rho\left(f_{n}-f\right) \rightarrow 0$ as $n \rightarrow \infty$. We write $f_{n} \rightarrow f(\rho)$.

(2) A sequence $\left(f_{n}\right)$ is said to be $\rho$-Cauchy if $\rho\left(f_{n}-f_{m}\right) \rightarrow 0$ as $n, m \rightarrow \infty$.

(3) $X_{\rho}$ is said to be complete if any $\rho$-Cauchy sequence is $\rho$-convergent.

(4) A subset $C \subset X_{\rho}$ is called $\rho$-closed if $f$ belongs to $C$ for any sequence $\left\{f_{n}\right\} \subset C$ with $f_{n} \rightarrow f(\rho)$.

(5) A subset $C \subset L_{\Phi, \rho}$ is called $\rho$-bounded if

$$
\delta_{\rho}(C)=\sup \{\rho(f-g): f, g \in C\}<+\infty,
$$

where $\delta_{\rho}(C)$ is said to be the $\rho$-diameter of $C$.

(6) We say the modular $\rho$ has Fatou property if

$$
\rho(f) \leq \liminf _{n \rightarrow \infty} \rho\left(f_{n}\right)
$$

whenever $f_{n} \rightarrow f(\rho)$. 
Let $C$ be a $\rho$-bounded subset of $X_{\rho}$. Then

(a) The $\rho$-Chebyshev radius of $C$ with respect to $f$ is defined by

$$
r_{\rho}(f, C)=\sup \{\rho(f-g): g \in C\} .
$$

(b) The $\rho$-Chebyshev radius of $C$ is defined by

$$
R_{\rho}(C)=\inf \left\{r_{\rho}(f, C): f \in C\right\} .
$$

(c) The $\rho$-Chebyshev center of $C$ is defined by

$$
Z_{\rho}(C)=\left\{f \in C: r_{\rho}(f, C)=R_{\rho}(C)\right\} .
$$

We say that $f \in C$ is a $\rho$-diametral point if $r_{\rho}(f, C)=\delta_{\rho}(C)$. The set $C$ is said to be $\rho$-diametral if each $f \in C$ is a $\rho$-diametral point.

We say that $X_{\rho}$ has $\rho$-normal structure if every nonempty $\rho$-bounded, $\rho$-closed, convex subset $C$ of $X_{\rho}$, not reduced to a single point, is not a $\rho$-diametral set.

We say that $X_{\rho}$ has property $(\mathrm{R})$ provided that every decreasing sequence $\left(C_{n}\right)$ of nonempty $\rho$-bounded, $\rho$-closed, convex subset of $X_{\rho}$ has nonempty intersection provided $C_{n} \neq \varnothing$ for any $n \in \mathbb{N}$.

For more details on Orlicz spaces we refer to [11, 8, 10, 9, 16, 6, 15, 20, 21, 29, 35. and for more details on modular spaces one can consult [23, 24, 25, 28, 30, 31, 32, 33, 34].

Let $C$ be a subset of $X_{\rho}$ and let $T: C \rightarrow C$ be a mapping. If there exists $k \in[0,1)$ such that

$$
\rho(T(f)-T(g)) \leq k \rho(f-g)
$$

for any $f, g \in C$, then the mapping $T$ is said to be $\rho$-contractive [23]. If the inequality

$$
\rho(T(f)-T(g)) \leq \rho(f-g)
$$

holds for any $f, g \in C$, then the mapping $T$ is said to be $\rho$-nonexpansive. $f \in C$ is called a fixed point of $T$ provided that $T(f)=f$. The family of the fixed points of $T$ is said to be the fixed point set and is denoted by Fix $T$. A subset $D \subset X_{\rho}$ is called $T$-invariant if $T(D) \subset D$.

2. Main results. We first show the notions of complex extreme points and complex strongly extreme points of Banach spaces to the modular spaces. For more details on these notions we refer to [3, 4, 2].

Definition $2.1([2])$. Let $X_{\rho}$ be a modular space. A point $x \in S\left(X_{\rho}\right)$ is said to be a complex extreme point of $B\left(X_{\rho}\right)$ if for any $y \in X_{\rho}$ with $y \neq 0$

$$
\sup _{|\lambda| \leq 1} \rho(x+\lambda y)>1 .
$$

$X_{\rho}$ is said to be complex strictly convex if every element of $S\left(X_{\rho}\right)$ is a complex extreme point of $B\left(X_{\rho}\right)$. 
Definition $2.2([2])$. Let $X_{\rho}$ be a modular space. A point $x \in S\left(X_{\rho}\right)$ is said to be a complex strongly extreme point of $B\left(X_{\rho}\right)$ if $\Delta_{c, \rho}(x, \varepsilon)>0$ for every $\varepsilon>0$, where

$$
\begin{aligned}
& \Delta_{c, \rho}(x, \varepsilon)=\inf \{1-|\lambda|: \lambda \in \mathbb{C}, 0<|\lambda| \leq 1, \\
& \left.\quad \text { and } \exists y \in X_{\rho}: \rho\left(x \pm \frac{y}{\lambda}\right) \leq \frac{1}{|\lambda|}, \rho\left(x \pm i \frac{y}{\lambda}\right) \leq \frac{1}{|\lambda|}, \rho(y) \geq \varepsilon\right\} .
\end{aligned}
$$

$X_{\rho}$ is said to be complex midpoint locally uniformly convex if every element of $S\left(X_{\rho}\right)$ is a complex strongly extreme point of $B\left(X_{\rho}\right)$.

THEOREM 2.3. Let $X_{\rho}$ be a modular space. If $x \in S\left(X_{\rho}\right)$ is a complex strongly extreme point of $B\left(X_{\rho}\right)$, then $x$ is a complex extreme point of $B\left(X_{\rho}\right)$.

Proof. Suppose that $x \in S\left(X_{\rho}\right)$ is not a complex extreme point of the closed unit ball $B\left(X_{\rho}\right)$. Then there exists $z \in X_{\rho} \backslash\{0\}$ such that

$$
\sup _{|\lambda| \leq 1} \rho(x+\lambda z) \leq 1 .
$$

Hence, we have

$$
\rho(x \pm z) \leq 1, \quad \rho(x \pm i z) \leq 1 .
$$

Letting $\varepsilon_{0}=\rho(z)>0$, we obtain $\Delta_{c, \rho}\left(x, \varepsilon_{0}\right)=0$ which is a contradiction.

The next result is an immediate corollary of the previous theorem.

COROLlary 2.4. Let $X_{\rho}$ be a modular space, if $X_{\rho}$ is complex midpoint locally uniformly convex, it is also complex strictly convex.

In order to prove that every Orlicz modular function space $L_{\Phi, \rho}$ is complex midpoint locally uniformly convex, we have first to recall a useful result ([4, p. 187]).

LEMmA 2.5. For any $\varepsilon>0$, there exists $\delta \in\left(0, \frac{1}{2}\right)$ such that if $u, v \in \mathbb{C}$ and

$$
|v| \geq \frac{\varepsilon}{8} \max _{e}|u+e v|
$$

then

$$
|u| \leq \frac{1-2 \delta}{4} \Sigma_{e}|u+e v|
$$

where

$$
\begin{gathered}
\max _{e}|u+e v|=\max \{|u+v|,|u-v|,|u+i v|,|u-i v|\}, \\
\Sigma_{e}|u+e v|=|u+v|+|u-v|+|u+i v|+|u-i v| .
\end{gathered}
$$

THEOREM 2.6. Let $L_{\Phi, \rho}$ be an Orlicz modular function space. Then $L_{\Phi, \rho}$ is complex midpoint locally uniformly convex.

Proof. Suppose that $x_{0} \in S\left(L_{\Phi, \rho}\right)$ is not a complex strongly extreme point of the unit ball $B\left(L_{\Phi, \rho}\right)$, by Definition 2.2 then there exists $\varepsilon_{0}>0$ such that $\triangle_{c, \rho}\left(x_{0}, \varepsilon_{0}\right)=0$. That is, there exist $\lambda_{n} \in \mathbb{C}$ with $\left|\lambda_{n}\right| \rightarrow 1$ and $y_{n} \in L_{\Phi, \rho}$ satisfying $\rho\left(y_{n}\right) \geq \varepsilon_{0}$, such that

$$
\rho\left(x_{0} \pm \frac{y_{n}}{\lambda_{n}}\right) \leq \frac{1}{\left|\lambda_{n}\right|}, \quad \rho\left(x_{0} \pm i \frac{y_{n}}{\lambda_{n}}\right) \leq \frac{1}{\left|\lambda_{n}\right|}
$$


for each $n \in \mathbb{N}$. Setting $z_{n}=y_{n} / \lambda_{n}$, we have

$$
\rho\left(z_{n}\right)=\rho\left(\frac{y_{n}}{\lambda_{n}}\right) \geq \rho\left(y_{n}\right) \geq \varepsilon_{0}
$$

and

$$
\rho\left(x_{0} \pm z_{n}\right) \leq \frac{1}{\left|\lambda_{n}\right|}, \quad \rho\left(x_{0} \pm i z_{n}\right) \leq \frac{1}{\left|\lambda_{n}\right|} .
$$

For the above $\varepsilon_{0}>0$, by Lemma 2.5 there exists $\delta_{0} \in\left(0, \frac{1}{2}\right)$, such that if $u, v \in \mathbb{C}$ and

$$
|v| \geq \frac{\varepsilon_{0}}{8} \max _{e}|u+e v|
$$

then

$$
|u| \leq \frac{1-2 \delta_{0}}{4} \Sigma_{e}|u+e v|
$$

For every $n \in \mathbb{N}$, we define

$$
\begin{gathered}
A_{n}=\left\{t \in T:\left|z_{n}(t)\right| \geq \frac{\varepsilon_{0}}{8} \max _{e}\left|x_{0}(t)+e z_{n}(t)\right|\right\}, \\
z_{n}^{(1)}: z_{n}^{(1)}(t)=z_{n}(t)\left(t \notin A_{n}\right), \quad z_{n}^{(1)}(t)=0\left(t \in A_{n}\right), \\
z_{n}^{(2)}: z_{n}^{(2)}(t)=0\left(t \notin A_{n}\right), \quad z_{n}^{(2)}(t)=z_{n}(t)\left(t \in A_{n}\right) .
\end{gathered}
$$

It is easy to see that $z_{n}=z_{n}^{(1)}+z_{n}^{(2)}$ for each $n \in \mathbb{N}$, and

$$
\begin{aligned}
\rho\left(z_{n}^{(1)}\right) & =\int_{T \backslash A_{n}} \Phi\left(\left|z_{n}(t)\right|\right) d \mu \leq \int_{T \backslash A_{n}} \Phi\left(\frac{\varepsilon_{0}}{8} \max _{e}\left|x_{0}(t)+e z_{n}(t)\right|\right) d \mu \\
& \leq \frac{\varepsilon_{0}}{8} \int_{T \backslash A_{n}} \Phi\left(\max _{e}\left|x_{0}(t)+e z_{n}(t)\right|\right) d \mu \\
& \leq \frac{\varepsilon_{0}}{8} \sum_{e} \rho\left(x_{0}+e z_{n}\right) \leq \frac{\varepsilon_{0}}{2\left|\lambda_{n}\right|}<\frac{3 \varepsilon_{0}}{4}
\end{aligned}
$$

for $n$ large enough since $\left|\lambda_{n}\right| \rightarrow 1$ as $n \rightarrow \infty$. Consequently, we deduce that

$$
\rho\left(z_{n}^{(2)}\right)>\frac{\varepsilon_{0}}{4}
$$

which shows that $\mu\left(A_{n}\right)>0$. Furthermore, we have

$$
\begin{aligned}
1 & =\rho\left(x_{0}\right)=\int_{A_{n}} \Phi\left(\left|x_{0}(t)\right|\right) d \mu+\int_{T \backslash A_{n}} \Phi\left(\left|x_{0}(t)\right|\right) d \mu \\
& \leq \int_{A_{n}} \Phi\left(\frac{1-2 \delta_{0}}{4} \Sigma_{e}\left|x_{0}(t)+e z_{n}(t)\right|\right) d \mu+\int_{T \backslash A_{n}} \Phi\left(\frac{1}{4} \Sigma_{e}\left|x_{0}(t)+e z_{n}(t)\right|\right) d \mu \\
& \leq\left(1-2 \delta_{0}\right) \int_{A_{n}} \Phi\left(\frac{1}{4} \Sigma_{e}\left|x_{0}(t)+e z_{n}(t)\right|\right) d \mu+\int_{T \backslash A_{n}} \Phi\left(\frac{1}{4} \Sigma_{e}\left|x_{0}(t)+e z_{n}(t)\right|\right) d \mu \\
& =\int_{T} \Phi\left(\frac{1}{4} \Sigma_{e}\left|x_{0}(t)+e z_{n}(t)\right|\right) d \mu-2 \delta_{0} \int_{A_{n}} \Phi\left(\frac{1}{4} \Sigma_{e}\left|x_{0}(t)+e z_{n}(t)\right|\right) d \mu \\
& \leq \frac{1}{4} \Sigma_{e} \int_{T} \Phi\left(\left|x_{0}(t)+e z_{n}(t)\right|\right) d \mu-2 \delta_{0} \int_{A_{n}} \Phi\left(\frac{1}{4} \Sigma_{e}\left|x_{0}(t)+e z_{n}(t)\right|\right) d \mu \\
& \leq \frac{1}{\left|\lambda_{n}\right|}-2 \delta_{0} \int_{A_{n}} \Phi\left(\frac{1}{4} \Sigma_{e}\left|x_{0}(t)+e z_{n}(t)\right|\right) d \mu .
\end{aligned}
$$


Notice that

$$
\int_{A_{n}} \Phi\left(\frac{1}{4} \Sigma_{e}\left|x_{0}(t)+e z_{n}(t)\right|\right) d \mu \geq \int_{A_{n}} \Phi\left(\left|z_{n}(t)\right|\right) d \mu=\rho\left(z_{n}^{(2)}\right)>\frac{\varepsilon_{0}}{4} .
$$

Hence,

$$
1=\rho\left(x_{0}\right) \leq \frac{1}{\left|\lambda_{n}\right|}-2 \delta_{0} \int_{A_{n}} \Phi\left(\frac{1}{4} \Sigma_{e}\left|x_{0}(t)+e z_{n}(t)\right|\right) d \mu<\frac{1}{\left|\lambda_{n}\right|}-\frac{\delta_{0} \varepsilon_{0}}{2} .
$$

Since $\left|\lambda_{n}\right| \rightarrow 1$ as $n \rightarrow \infty$, letting $n \rightarrow \infty$ we get a contradiction

$$
1=\rho\left(x_{0}\right)<\frac{1}{\left|\lambda_{n}\right|}-\frac{\delta_{0} \varepsilon_{0}}{2}<1,
$$

which completes the proof.

Combining Corollary 2.4 and Theorem 2.6 we obtain immediately the following result. COROLlary 2.7. Let $L_{\Phi, \rho}$ be an Orlicz modular function space. Then $L_{\Phi, \rho}$ is complex strictly convex.

Now we study the problem of existence of fixed points for mean nonexpansive mappings in the modular sense in Orlicz modular function spaces. Before we state the fixed point theorem, we have to introduce the concept of mean nonexpansive mappings in the modular sense.

Definition 2.8. Let $C$ be a subset of $X_{\rho}$ and let $T: C \rightarrow C$ be a mapping. We say that $T$ is a mean nonexpansive mapping in the modular sense provided that

$$
\rho(T(f)-T(g)) \leq a \rho(f-g)+b \rho(f-T(g)),
$$

where $a, b \geq 0$ and $a+b \leq 1$.

Next, we generalize the results for nonexpansive mappings in the modular sense ([22, Theorem 3.10]) to mean nonexpansive mappings in the modular sense.

THEOREM 2.9. Let $C \subset L_{\Phi, \rho}$ be a nonempty, $\rho$-closed, $\rho$-bounded, convex subset and let $T: C \rightarrow C$ be a mean nonexpansive mapping in the modular sense. Then there exists $D \subset C$, which is nonempty, $\rho$-closed, convex and T-invariant, such that

$$
\delta_{\rho}(D) \leq \frac{1}{2} \delta_{\rho}(C)+\frac{1}{2} R_{\rho}(C) .
$$

Proof. If $R_{\rho}(C)=\delta_{\rho}(C)$ then we can take $D=C$. Assume therefore that $R_{\rho}(C)<\delta_{\rho}(C)$. Let

$$
\gamma=\frac{1}{2} \delta_{\rho}(C)+\frac{1}{2} R_{\rho}(C)
$$

Since $R_{\rho}(C)<\gamma$, there exists $f \in C$ such that $r_{\rho}(f, C) \leq \gamma$. Define the family

$$
\mathfrak{F}=\{E \subset C: T(E) \subset E, f \in E, E \text { is } \rho \text {-closed and convex }\}
$$

and observe that $\mathfrak{F} \neq \varnothing$ since $C \in \mathfrak{F}$. Let $D=\bigcap_{E \in \mathfrak{F}} E$ and notice that $D \subset C, f \in D$, $D$ is also $\rho$-closed and convex and $T(D) \subset D$. Let us define the $\rho$-balls for all $\alpha>0$ by

$$
B_{\rho}(g, \alpha)=\left\{h \in L_{\Phi, \rho}: \rho(g-h) \leq \alpha\right\}, \quad g \in L_{\Phi, \rho} .
$$


Then we observe that every $\rho$-ball $B_{\rho}(g, \alpha)$ is convex and $\rho$-closed by Fatou's lemma. Let $\mathfrak{G}$ be a family of all $\rho$-balls which contain $T(D) \cup\{f\}$. The convex hull of $T(D) \cup\{f\}$ is denoted by conv $(T(D) \cup\{f\})$. It is not difficult to see that

$$
\operatorname{conv}(T(D) \cup\{f\})=\bigcap_{B \in \mathfrak{G}} B \cap D
$$

and the set conv $(T(D) \cup\{f\})$ is $\rho$-closed, convex and contains $f$. We will prove that it is also $T$-invariant. Indeed, let $F=\operatorname{conv}(T(D) \cup\{f\})$ and notice that $F \subset D$. Then

$$
T(F) \subseteq T(D) \subseteq F
$$

which implies $F=D$. Let

$$
D_{r}=\left\{h \in D: r_{\rho}(h, D) \leq r\right\},
$$

then $f \in D_{r}$ since $r_{\rho}(f, C) \leq r$. Notice that

$$
D_{r}=\bigcap_{g \in D} B_{\rho}(g, r) \cap D .
$$

Hence, $D_{r}$ is convex and $\rho$-closed. Let us prove that $D_{r}$ is $T$-invariant. Take $h \in D_{r}$, then $h \in D$. In view of the definition of $D_{r}$, we have $D \subset B_{\rho}(h, r)$. For any $z \in T(D) \subset D$, there exists $g \in D$ such that $T(g)=z$. Then

$$
\begin{aligned}
\rho(T(h)-z) & =\rho(T(h)-T(g)) \leq a \rho(h-g)+b \rho(h-T(g)) \\
& \leq a r+b \rho(h-T(g)) \leq(a+b) r \leq r
\end{aligned}
$$

which implies $T(D) \subset B_{\rho}(T(h), r)$. On the other hand, $f$ belongs to $B_{\rho}(T(h), r)$ since $r_{\rho}(f, C) \leq r$ and $T(h) \in D$. Thus

$$
D=F \subset B_{\rho}(T(h), r),
$$

which implies $r_{\rho}(T(h), D) \leq r$. Then $T(h) \in D_{r}$, we obtain $T\left(D_{r}\right) \subseteq D_{r}$. Consequently, $D_{r} \in \mathfrak{F}$ and by the definition of $D$, we have $D=D_{r}$. Hence, $\delta_{\rho}(D) \leq r$ which shows that $D$ is the desired set.

TheOREM 2.10. Suppose that $L_{\Phi, \rho}$ has property $(\mathrm{R})$ and $\rho$-normal structure. Let $C \subset L_{\Phi, \rho}$ be a nonempty, $\rho$-closed, $\rho$-bounded, convex subset and let $T: C \rightarrow C$ be a mean nonexpansive mapping in the modular sense. Then the mapping $T$ has a fixed point in $C$.

Proof. Let $\mathfrak{A}$ be a family of all nonempty, $\rho$-closed, convex and $T$-invariant subsets of $C$. Define $\delta_{0}: \mathfrak{A} \rightarrow \mathbb{R}^{+}$by the formula

$$
\delta_{0}(A)=\inf \left\{\delta_{\rho}(B): B \in \mathfrak{A}, B \subset A\right\} .
$$

Let $\varepsilon_{n}>0$ for each $n \in \mathbb{N}$ and $\varepsilon_{n} \rightarrow 0$ as $n \rightarrow \infty$. Then we can find a decreasing sequence $\left\{A_{n}\right\}$ satisfying $A_{n} \in \mathfrak{A}$ and $\delta_{\rho}\left(A_{n+1}\right) \leq \delta_{0}\left(A_{n}\right)+\varepsilon_{n}$. Since $L_{\Phi, \rho}$ has property (R), we have $A_{0}=\bigcap_{n \in \mathbb{N}} A_{n} \neq \varnothing$. Clearly, $A_{0} \in \mathfrak{A}$. It remains to show that $A_{0}$ has only one point. Indeed, by Theorem 2.9 , there exists $D \in \mathfrak{A}$ with $D \subset A_{0}$ such that

$$
\delta_{\rho}(D) \leq \frac{1}{2}\left(\delta_{\rho}\left(A_{0}\right)+R_{\rho}\left(A_{0}\right)\right) .
$$


Since $D \subset A_{n}$ for each $n \in \mathbb{N}$, it follows that $\delta_{0}\left(A_{n}\right) \leq \delta_{\rho}(D)$. Hence,

$$
\delta_{\rho}(D) \leq \delta_{\rho}\left(A_{0}\right) \leq \delta_{\rho}\left(A_{n+1}\right) \leq \delta_{0}\left(A_{n}\right)+\varepsilon_{n} \leq \delta_{\rho}(D)+\varepsilon_{n},
$$

which implies that

$$
\delta_{\rho}(D) \leq \delta_{\rho}\left(A_{0}\right) \leq \delta_{\rho}(D)+\varepsilon_{n} .
$$

Letting $n \rightarrow \infty$, we obtain $\delta_{\rho}(D)=\delta_{\rho}\left(A_{0}\right)$. By combining with inequality (1), it follows that

$$
\delta_{\rho}\left(A_{0}\right) \leq R_{\rho}\left(A_{0}\right)
$$

Since always $\delta_{\rho}\left(A_{0}\right) \geq R_{\rho}\left(A_{0}\right)$, we obtain $\delta_{\rho}\left(A_{0}\right)=R_{\rho}\left(A_{0}\right)$. Since $L_{\Phi, \rho}$ has $\rho$-normal structure, we deduce that $A_{0}$ is reduced to a single point. Since $A_{0}$ is $T$-invariant, this point is a fixed point for $T$.

Acknowledgments. This work was supported by Training Program for Youth Innovation Talents of Heilongjiang Educational Committee under Grant UNPYSCT-2017078, Postdoctoral Science Foundation of Heilongjiang Province under Grant LBH-Q18067 and the Fundamental Research for Universities of Heilongjiang Province under Grant LGYC2018JQ001.

\section{References}

[1] O. Blasco, M. Pavlović, Complex convexity and vector-valued Littlewood-Paley inequalities, Bull. Lond. Math. Soc. 35 (2003), 749-758.

[2] L. Chen, D. Chen, Y. Jiang, Complex convexity of Orlicz modular sequence spaces, J. Funct. Spaces 2016, Art. ID 5917915, 6 pp.

[3] L. Chen, Y. Cui, H. Hudzik, Criteria for complex strongly extreme points of Musielak-Orlicz function spaces, Nonlinear Anal. 70 (2009), 2270-2276.

[4] S. Chen, Geometry of Orlicz Spaces, Dissertationes Math. (Rozprawy Mat.) 356 (1996).

[5] S. Chen, H. Hudzik, W. Kowalewski, Y. Wang, M. Wisła, Approximative compactness and continuity of metric projector in Banach spaces and applications, Sci. China Ser. A 51 (2008), 293-303.

[6] S. Chen, X. He, H. Hudzik, Monotonicity and best approximation in Banach lattices, Acta Math. Sin. (Engl. Ser.) 25 (2009), 785-794.

[7] C. Choi, A. Kamińska, H. J. Lee, Complex convexity of Orlicz-Lorentz spaces and its applications, Bull. Pol. Acad. Sci. Math. 52 (2004), 19-38.

[8] Y. Cui, L. Duan, H. Hudzik, M. Wisła, Basic theory of p-Amemiya norm in Orlicz spaces $(1 \leq p \leq \infty)$ : extreme points and rotundity in Orlicz spaces endowed with these norms, Nonlinear Anal. 69 (2008), 1796-1816.

[9] Y. Cui, H. Hudzik, L. Ji, M. Wisła, Strongly extreme points in Orlicz spaces equipped with the p-Amemiya norm, Nonlinear Anal. 71 (2009), 6343-6364.

[10] Y. Cui, H. Hudzik, R. Kumar, L. Maligranda, Composition operators in Orlicz spaces, J. Aust. Math. Soc. 76 (2004), 189-206.

[11] Y. Cui, H. Hudzik, R. Płuciennik, Extreme points and strongly extreme points in Orlicz spaces equipped with the Orlicz norm, Z. Anal. Anwend. 22 (2003), 789-817.

[12] M. M. Czerwińska, A. Kamińska, Complex rotundities and midpoint local uniform rotundity in symmetric spaces of measurable operators, Studia Math. 201 (2010), 253-285. 
[13] M. M. Czerwińska, A. Parrish, Complex extreme points in Marcinkiewicz spaces, Positivity 19 (2015), 121-135.

[14] W. Davis, D. Darling, N. Tomczak-Jaegermann, The complex convexity for quasi-normed linear spaces, J. Funct. Anal. 55 (1984), 110-150.

[15] P. N. Dowling, Z. B. Hu, D. Mupasiri, Complex convexity in Lebesgue-Bochner function spaces, Trans. Amer. Math. Soc. 348 (1996), 127-139.

[16] P. Foralewski, H. Hudzik, R. Płuciennik, Orlicz spaces without extreme points, J. Math. Anal. Appl. 361 (2010), 506-519.

[17] J. Globevnik, On complex strict and uniform convexity, Proc. Amer. Math. Soc. 47 (1975), $175-178$.

[18] K. Goebel, S. Reich, Uniform Convexity, Hyperbolic Geometry, and Nonexpansive Mappings, Monogr. Textbooks Pure Appl. Math. 83, Marcel Dekker, New York, 1984.

[19] H. Hudzik, A. Narloch, Relationships between monotonicity and complex rotundity properties with some consequences, Math. Scand. 96 (2005), 289-306.

[20] A. Kamińska, On uniform convexity of Orlicz space, Indag. Math. 44 (1982), 27-36.

[21] A. Kamińska, On some convexity properties of Musielak-Orlicz spaces, Rend. Circ. Mat. Palermo (2) Suppl., no. 5 (1984), 63-72.

[22] M. A. Khamsi, W. M. Kozłowski, S. Chen, Some geometrical properties and fixed point theorems in Orlicz spaces, J. Math. Anal. Appl. 155 (1991), 393-412.

[23] M. A. Khamsi, W. M. Kozłowski, S. Reich, Fixed point theory in modular function spaces, Nonlinear Anal. 14 (1990), 935-953.

[24] W. A. Kirk, Nonexpansive mappings in metric and Banach spaces, Rend. Sem. Mat. Fis. Milano 51 (1981), 133-144.

[25] W. M. Kozłowski, Modular Function Spaces, Monogr. Textbooks Pure Appl. Math. 122, Marcel Dekker, New York, 1988.

[26] H. J. Lee, Monotonicity and complex convexity in Banach lattices, J. Math. Anal. Appl. 307 (2005), 86-101.

[27] H. J. Lee, Complex convexity and monotonicity in quasi-Banach lattices, Israel J. Math. 159 (2007), 57-91.

[28] P. D. Liu, Y. Hou, Geometry of complex Banach spaces, Adv. Math. (China) 27 (1998), $1-20$.

[29] L. Maligranda, Orlicz Spaces and Interpolation, Seminários de Matemática 5, Univ. of Campinas, Campinas SP, Brazil, 1989.

[30] J. Musielak, Orlicz Spaces and Modular Spaces, Lecture Notes in Math. 1034, Springer, Berlin, 1983.

[31] J. Musielak, W. Orlicz, On modular spaces, Studia Math. 18 (1959), 49-65.

[32] J. Musielak, W. Orlicz, Some remarks on modular spaces, Bull. Acad. Polon. Sci. Sér. Sci. Math. Astr. Phys. 7 (1959), 661-668.

[33] H. Nakano, Modular Semi-Ordered Linear Spaces, Maruzen Co. Ltd., Tokyo, 1950.

[34] W. Orlicz, A note on modular spaces, Bull. Acad. Polon. Sci. Sér. Sci. Math. Astr. Phys. 9 (1961), 157-162.

[35] M. Rao, Z. Ren, Theory of Orlicz Spaces, Monogr. Textbooks Pure Appl. Math. 146, Marcel Dekker, New York, 1991.

[36] S. Reich, D. Shoikhet, Nonlinear Semigroups, Fixed Points, and Geometry of Domains in Banach Spaces, Imperial College Press, London, 2005.

[37] E. Thorp, R. Whitley, The strong maximum modulus theorem for analytic functions into a Banach space, Proc. Amer. Math. Soc. 18 (1967), 640-646. 